PSICOLOGÍA

IBEROAMERICANA
Psicología Iberoamericana ISSN: 1405-0943

revista.psicologia@ibero.mx

Universidad Iberoamericana, Ciudad de México México

\title{
Paternidad en adolescentes en conflicto con la ley: Historias de vida
}

Toiber Rodríguez, Marla Naiví; Mancillas Bazán, Celia; Montero Pardo, Xolyanetzin; Ramos Beltrán, Ignacio

Paternidad en adolescentes en conflicto con la ley: Historias de vida

Psicología Iberoamericana, vol. 27, núm. 2, 2019

Universidad Iberoamericana, Ciudad de México, México

Disponible en: http://www.redalyc.org/articulo.oa?id=133962309002 


\title{
Paternidad en adolescentes en conflicto con la ley: Historias de vida
}

\author{
Marla Naiví Toiber Rodríguez naivi17@hotmail.com \\ Secretaria de Salud, México \\ http://orcid.org/0000-0001-8100-4297 \\ Celia Mancillas Bazán \\ Universidad Iberoamericana, México \\ http://orcid.org/0000-0002-6833-7959 \\ Xolyanetzin Montero Pardo \\ Universidad Autónoma de Sinaloa, México \\ http://orcid.org/0000-0003-3656-0942 \\ Ignacio Ramos Beltrán \\ Universidad Nacional Autónoma de México, México \\ http://orcid.org/0000-0002-6024-4766
}

Psicología Iberoamericana, vol. 27, núm. 2, 2019

Universidad Iberoamericana, Ciudad de México, México

Recepción: 04 Marzo 2019 Aprobación: 13 Noviembre 2019

Redalyc: http://www.redalyc.org/ articulo.oa?id=133962309002
Resumen: El propósito de este artículo es analizar la construcción de la paternidad de adolescentes en conflicto con la ley. Por lo anterior, se realizó un estudio exploratorio y cualitativo; se construyeron historias de vida a través de la técnica de entrevista a profundidad. La muestra se conformó por 3 adolescentes en conflicto con la ley. Con la información obtenida se llevó a cabo un análisis de contenido interpretativo mediante el cual se obtuvieron 5 categorías: 1) experiencias en la familia de origen; 2) conductas de riesgo y delictivas; 3) curso de la paternidad: embarazo y nacimiento de su hijo; 4) transmisión intergeneracional de la violencia; y 5) familia reconstituida. En los discursos de los participantes se reconoce un transitar entre sus propias experiencias como hijos y la decisión de interrumpir la transmisión intergeneracional de la violencia y carencia afectiva. El estudio es una contribución al análisis de la paternidad, no sólo biológica sino también adquirida, lo que implica un elemento de mayor complejidad al brindar tratamiento psicoterapéutico a los padres adolescentes en conflicto con la ley que se encuentran en proceso de reinserción social.

Palabras clave: paternidad adolescente, historia de vida, adolescente en conflicto con la ley, metodología cualitativa, familia reconstituida.

Abstract: The main aim of this article is to understand how adolescents in conflict with the law construct their ideas around fatherhood. To explore this issue, an exploratory and qualitative study was carried out. Life stories were constructed through in-depth interviews. The sample consisted of 3 adolescents in conflict with the law. Through the interpretative content analysis, we obtained the following categories: 1) family of origin experiences, 2) risk and criminal conduct, 3) trajectory of fatherhood: pregnancy and child's birth, 4) intergenerational transmission of violence, and 5) reconstituted families. Participants' discourses included a trajectory of their own experiences as children and the decision to prevent the intergenerational transmission of violence and emotional deprivation. Within the reconstituted families that these adolescents established, their main parental role was to replace the biological father. This role can promote rigid beliefs and patterns of interaction with the intention of conceiving themselves as a nuclear family without considering previous family history. This research is a contribution to the study of fatherhood, not only biological but also acquired. It will also contribute to the field of psychotherapeutic treatment for adolescent parents in conflict with the law, who are in the process of social reintegration. 
Keywords: adolescent fatherhood, life story, adolescent in conflict with the law, qualitative methodology, reconstituted family.

\section{Introducción}

El embarazo adolescente es un fenómeno que se encuentra en la agenda pública de México porque tiene una alta incidencia y múltiples consecuencias, por ejemplo, la deserción escolar de las adolescentes, rechazo y exclusión social. Por parte de los hombres, se pueden mostrar desvinculados del embarazo o las barreras familiares, institucionales y sociales les pueden impedir ejercer su paternidad (Olvera, 2012). De acuerdo al Instituto Nacional de Estadística y Geografía (INEGI, 2015) en México el $18.4 \%$ de la población está conformada por adolescentes entre 10 y 19 años de edad. Entre 2009 y 2014 el embarazo adolescente ha aumentado un 10\%. En países como Colombia, Perú y México, la investigación y los programas psicosociales destinados a los padres adolescentes son escasos porque el centro de interés ha sido la madre adolescente (De Keijzer, 2003).

En México existen ciertas características del entorno sociocultural donde hay mayor propensión a que ocurra el embarazo adolescente, como ser pobre, vivir con un padrastro, ser mujer en una sociedad patriarcal, tener baja escolaridad, ser menor de edad y tener muchos hermanos (Stern, 2004). En Estados Unidos se identificó que también influyen las características del ambiente social, la posición en la estructura familiar, el estrés parental, la actividad sexual temprana, el grupo de pares y las conductas desviadas (Thornberry, Wei, Stouthamer-Loeber, \& Van Dyke, 2000).

Algunos factores de riesgo en el contexto familiar que favorecen las conductas delictivas son el rechazo parental, tener un proceso inadecuado de socialización (López \& López, 2008), con menor supervisión o con exceso de control (Mestre, Samper, \& Frías, 2004), ser maltratados (Smith, Ireland, \& Thornberry, 2005), experimentar estrés derivado de un nivel socioeconómico bajo, las prácticas de crianza (Chavarín-García \& Gálvez-Hernández, 2018), el estilo de comunicación y la falta de disciplina en el hogar (Lykken, 2000). De igual forma, cometer delitos en la adolescencia es un factor de riesgo alto para convertirse en padre adolescente (Thornberry et al., 2000).

La paternidad se entiende como un campo de prácticas y significaciones culturales y sociales en torno a la reproducción, vínculo y cuidado de los hijos. Los comportamientos parentales varían según diversos elementos como el ciclo vital de las personas, la relación con la co-genitora de su hijo, el apoyo por parte de sus redes familiares y las consecuencias de la paternidad en su proyecto de vida (Fuller, 2000).

Los varones de nivel socioeconómico medio de la Ciudad de México definen la paternidad como una oportunidad de aprender y recrearse con la crianza. Experimentan una confrontación entre los estereotipos masculinos porque tratan de ser padres cercanos, afectivos y comprometidos con sus hijos, pero esto es silenciado. Cuando integran en 
la subjetividad el deseo, planeación y decisión de los hijos como parte de su proyecto de vida, viven la paternidad como algo extraordinario que llega a cambiar la existencia de algunos varones (Salguero, 2006). En jóvenes mexicanos, la paternidad se continúa relacionando con características como ejercer la autoridad, la responsabilidad y ser proveedor, pero en un nivel menor al que se ha establecido tradicionalmente. Conciben a la paternidad responsable como una integración de la esfera económica, afectiva y de crianza (Balam, Valdez, Ramírez, \& Padilla, 2018). La responsabilidad se considera como uno de los constructos que definen el desempeño de ser un buen padre, la cual implica generar protección económica, el cuidado, la atención de los hijos y de la pareja (De JesúsReyes \& Cabello-Garza, 2011). Es importante estudiar la paternidad de adolescentes mexicanos que no tienen claro un proyecto de vida, porque ésta puede proporcionarles reconocimiento social y familiar (De JesúsReyes \& Cabello-Garza, 2011). Así mismo, es importante considerar que los factores socioculturales, el estrato social, el sector ocupacional y la generación marcan diferencias importantes en la participación de los hombres en la crianza de sus hijos (Franzoni, 2014).

Aunque tradicionalmente se ha estudiado la paternidad desde el punto de vista de la irresponsabilidad y la poca participación de los hombres en la crianza de sus hijos (Shepard, 2000), en la última década se ha incrementado el interés hacia el estudio de la paternidad (Balam et al., 2018). Por ejemplo, en Nueva Zelanda han comenzado a llevarse a cabo estudios en los que se relaciona la paternidad adolescente como una oportunidad de cambio en el curso de la vida o como un punto de inflexión (Tuffin, Rouch, \& Frewin, 2010). En Estados Unidos hay más investigaciones ahora sobre la influencia de la paternidad en la trayectoria criminal de los adolescentes (Shannon \& Abrams, 2007), la forma como asumen la responsabilidad de sus hijos (Ngu \& Florsheim, 2011) o la reparación intergeneracional que quieren hacer de sus propias vidas a partir de su rol parental (Landers, Mitchell, \& Coates, 2015).

En la población de padres adolescentes descendientes de mexicanos, se ha identificado que intentan proteger a sus hijos de tener una vida similar a la que ellos tuvieron, es decir, haber experimentado violencia familiar, usado drogas, vivir en la calle o pertenecer a pandillas. Lo que buscan es proveer a sus hijos de amor y apoyo aunque ello implique romper los estereotipos sociales de su rol como padres (Parra-Cardona, Sharp, \& Wampler, 2008).

En Madrid, España, los estudios sobre las familias reconstituidas demuestran que existe una concepción estática de la familia porque no integra las dimensiones temporal y espacial en el sentido de los tiempos que conviven con cada uno de sus padres y el lugar en el que lo hacen, ya que los hijos circulan entre los hogares paternos y maternos (Jociles $\&$ Villaamil, 2008). Los padres se pueden encontrar con ambigüedad de su rol, conflicto coparental y desacuerdos con la pareja acerca del parentaje (Jensen, 2019). En Estados Unidos, Papernow (2018) considera que se requieren investigaciones de familias reconstituidas encabezadas 
por parejas jóvenes, no casadas, con bajo nivel educativo, bajos ingresos económicos y que han cursado por algún encarcelamiento.

Los términos de familia reconstituida, ensamblada, adoptiva han sido ampliamente utilizados para nombrar a una familia en donde al menos uno de los adultos tiene uno o varios hijos de una relación previa (Ganong \& Coleman, 2018). En Italia, Fruggeri (2016) menciona que el término familia reconstituida considera que el padre a cargo, la nueva pareja y los hijos, están buscando reintegrarse al modelo nuclear familiar y no tienen en cuenta a la separación como un evento estructurante de las relaciones y la historia familiar, por ello recomienda emplear el término familia reconformada. También propone el término "hijo adquirido" (Fruggeri, 2001) para nombrar la relación entre padre no biológico-hijo de la pareja, en lugar del término hijastro, porque este último puede tener una connotación negativa.

El objetivo del presente estudio es analizar la forma como los padres adolescentes en conflicto con la ley construyen su paternidad de acuerdo con lo que experimentaron en su niñez dentro de su familia de origen. Los objetivos específicos son describir el contexto que favoreció que el adolescente comenzara a delinquir, la forma en que la paternidad influye en continuar o no con dicha conducta y la relación que han desarrollado con su hijo adquirido.

El contexto en el que se realizó la investigación fue la Comunidad Externa de Atención para Adolescentes (CEAA) de la Dirección General de Tratamiento para Adolescentes de la Subsecretaria del Sistema Penitenciario del Gobierno de la Ciudad de México. La CEAA es una comunidad especializada en brindar diagnóstico y tratamiento a adolescentes en modalidad de libertad asistida. Ésta consiste en la obligación del adolescente a someterse a la vigilancia y supervisión del personal especializado que determine la Autoridad Ejecutora, a través de un Programa diseñado para tal efecto (Honorable Congreso de la Unión, 2007).

\section{Método}

En el estudio se empleó la metodología cualitativa para responder a la pregunta de investigación: ¿̨cuál es la forma como los padres adolescentes en conflicto con la ley construyen su paternidad de acuerdo con lo que experimentaron en su niñez dentro de su familia de origen? Los objetivos específicos son: describir el contexto que favoreció que el adolescente comenzara a delinquir, la forma como la paternidad influye en continuar o no dicha conducta y la relación que han desarrollado con su hijo adquirido. Se realizaron entrevistas en profundidad con 3 participantes en la Ciudad de México. Para analizar las historias de vida se llevó a cabo un análisis de contenido interpretativo. 


\section{Participantes}

La selección de los participantes se llevó a cabo de forma propositiva. Se contó con la colaboración de 3 padres adolescentes en conflicto con la ley que al menos tuvieran un hijo adquirido. El estudio se llevó a cabo en la Ciudad de México. Para tener una visión más detallada de las características sociodemográficas de los participantes, ver la Tabla 1.

\section{Tabla 1}

Descripción sociodemográfica de los participantes

\section{Tabla 1}

Descripción sociodemográfica de los participantes

\begin{tabular}{|c|c|c|c|c|c|c|}
\hline Nombre & Edad & $\begin{array}{c}\text { Estado } \\
\text { civil }\end{array}$ & Delito & Escolaridad & $\begin{array}{l}\text { Alcaldía o } \\
\text { entidad de } \\
\text { procedencia }\end{array}$ & $\begin{array}{c}\text { No. } \\
\text { Hijos/ } \\
\text { hijo } \\
\text { adquirido }\end{array}$ \\
\hline Bruno & $\begin{array}{c}18 \\
\text { años }\end{array}$ & $\begin{array}{l}\text { unión } \\
\text { libre }\end{array}$ & $\begin{array}{c}\text { robo } \\
\text { agravado }\end{array}$ & $\begin{array}{l}\text { secundaria } \\
\text { en curso }\end{array}$ & Cuauhtémoc & $\begin{array}{c}1 \text { hija/ } 1 \\
\text { hijo } \\
\text { adquirido }\end{array}$ \\
\hline Hugo & $\begin{array}{c}17 \\
\text { años }\end{array}$ & $\begin{array}{l}\text { unión } \\
\text { libre }\end{array}$ & $\begin{array}{c}\text { robo } \\
\text { calificado }\end{array}$ & $\begin{array}{l}\text { secundaria } \\
\text { trunca }\end{array}$ & Cuauhtémoc & $\begin{array}{c}1 \text { hijo/ } 1 \\
\text { hija } \\
\text { adquirida }\end{array}$ \\
\hline Carlos & $\begin{array}{c}18 \\
\text { años }\end{array}$ & $\begin{array}{l}\text { unión } \\
\text { libre }\end{array}$ & $\begin{array}{c}\text { robo } \\
\text { calificado }\end{array}$ & $\begin{array}{l}\text { secundaria } \\
\text { trunca }\end{array}$ & $\begin{array}{l}\text { Estado de } \\
\text { México }\end{array}$ & $\begin{array}{c}1 \text { hijo/ } 1 \\
\text { hijo } \\
\text { adquirido }\end{array}$ \\
\hline
\end{tabular}

Los criterios de inclusión fueron: a) el rango de edad entre 15 y 18 años de edad; b) ser padres biológicos; c) el tipo de delito era robo agravado y calificado; d) cumplir con una medida alterna por la comisión de un delito. Cabe aclarar que algunos adolescentes cumplen su mayoría de edad mientras están cumpliendo la medida alterna impuesta por el juez y por tal motivo se les incluyó en el estudio.

\section{Las entrevistas}

Se empleó la técnica de entrevista en profundidad semiestructuradas (Rodríguez, Gil, \& García, 1999). Las historias de vida se conformaron a partir de la frase: "Cuéntame la historia de tu vida". Los temas guía fueron: la comisión del delito y el consumo de drogas, la familia de origen, la relación de pareja, el embarazo y la paternidad. La entrevista a profundidad se caracteriza por su carácter holístico o en un solo acto 
y el grado de dirección-no dirección con que se desarrolla (Vela, 2008). En la entrevista emergen hechos bisagras o puntos de inflexión en los que es posible determinar un antes y un después en la vida del participante (Mallimaci \& Giménez, 2006).

El estudio se llevó a cabo en las instalaciones de la Comunidad Externa de Atención para Adolescentes (CEAA), de la Ciudad de México. Se condujo de forma ética ya que los participantes aceptaron participar y firmaron una carta de consentimiento en donde se estipulaba la confidencialidad, el anonimato y su participación de forma voluntaria en la investigación.

\section{Análisis}

Las entrevistas se audiograbaron, se transcribieron y se empleó el software Atlas.ti v.7 como herramienta para generar códigos y categorías principales. Los nombres de los adolescentes y sus familiares han sido cambiados para proteger su confidencialidad. Finalmente se realizó un análisis interpretativo de contenido el cual consistió en los siguientes pasos: 1) se leyeron todos los datos para lograr la inmersión y obtener una idea del todo; 2) se leyó palabra por palabra para derivar códigos; 3) se tomaron notas de las impresiones, pensamientos y análisis inicial del investigador; 4) se codificó y se identificaron categorías emergentes para agrupar códigos; y 5) se definió cada categoría, subcategoría y código (Hsieh \& Shannon, 2005).

\section{Resultados}

Para contestar a la pregunta de investigación respecto a analizar la forma como los padres adolescentes en conflicto con la ley construyen su paternidad, se realizó un análisis de contenido horizontal. Éste se conformó por la codificación y homologación de códigos. Se construyeron categorías de análisis de las historias de vida de acuerdo con los siguientes eventos: experiencias de la niñez dentro de su familia de origen, el contexto para comenzar a delinquir, influencia de paternidad en la conducta delictiva y la relación que han desarrollado con su hijo adquirido. Dicho análisis contrasta los discursos individuales desde las categorías de análisis por cada participante y a partir de ellas se obtuvieron las siguientes categorías principales: 1) experiencias en la familia de origen; 2) conductas de riesgo y delictivas; 3 ) curso de la paternidad: embarazo y nacimiento de su hijo. Se obtuvieron dos categorías emergentes: 4) transmisión intergeneracional de la violencia y 5) familia reconstituida.

A continuación, se explora cada una de las categorías y los códigos que las conforman. 
Se refiere a la vivencia a lo largo de la vida del adolescente de situaciones como la violencia familiar, abandono, muerte de su padre o de su figura paterna. Y la actual relación que mantienen con su familia de origen, si la conciben como red de apoyo o como un estresor en su vida.

La figura del padre de los adolescentes va en un continuum donde en un polo se encuentra la ausencia, después la presencia con el uso de violencia y en el otro polo la cercanía afectiva y el rol de autoridad flexible.

Padre con rol ausente: "No pues a mi papá ni lo conozco.... [mi mamá] me dice que no sabe y ya mejor ni le digo.... me hizo falta su cariño de chico, pero ahorita ya no... [mi abuelo] fue el que vio por nosotros, fue al que agarramos como papá (...) era regañón, mandón, pero también veía mucho por nosotros" (Carlos).

Padre con rol presente y violento: "tuvimos un papá que pues... ahora sí que nos maltrataba, nos daba unas friegas por no hacer las cosas bien.... si lo quiero (...) pero asi como un tipo de amor o de afecto hacia él no"(Bruno).

Padre con rol flexible: "mi papá era policía pues me manejaba también... siempre me consentia... me gustaba mucho estar con él (...) cuando tenía 13 años murió, dicen que de la influenza" (Hugo).

\section{Conductas de riesgo y delictivas}

Se refiere a los elementos que facilitaron que el adolescente iniciara las conductas de riesgo como desertar de la escuela, la violencia familiar, la adultización precoz y convivir con pares que delinquen.

"A los 11 o 12 años empecé a irme con las malas amistades, los amigos, las drogas, el alcohol. Después, entre los 14 y 15 años vendia droga, todo eso, andaba en el desmadre ¿no?"... a los 15 años “intenté irme por la derecha (...) y no la veía llegar (...) y fue el secuestro y a la vez valió la pena y a la vez no, si fue dinero y todo eso, pero también perder un poco de tiempo, de ver a mi gordo, de verlo crecer" (Bruno).

"Es que estaba con unos amigos, yo pensaba que no eran malos y esos chavos traian pistola y eso ¿no?, y estábamos tomando y traía el coche yo iba manejando y se bajaron en un parque abi y robaron y como yo iba manejando pues dijeron que yo también habia robado" (Hugo).

"Deje de ir [a la escuela] desde de segundo de secundaria, ya no me gustó y mi mamá ya no me inscribió ¿para qué iba a gastar? si ya no me gustó" (Carlos).

\section{Curso de la paternidad: embarazo y nacimiento de su bijo}

Se refiere a la forma como experimentaron la noticia del embarazo, el involucramiento que tuvieron durante éste y el nacimiento de su hijo. Este último evento les permitió reflexionar acerca de sus propias experiencias como hijos y considerar formas diferentes de satisfacer las necesidades 
instrumentales y afectivas de los suyos. También se identificó la función que tiene la paternidad para continuar o no con la conducta delictiva.

En el caso de Bruno durante el embarazo él seguía "en el desmadre", pues "no me caía el veinte (...) aunque eso sí (...) de lo que ganaba de vender droga, de andar robando, ella en su embarazo la pasó bien, no le faltó estudios, nada". El nacimiento de su hija marcó el momento donde "me cayó el veinte (...) es una emoción muy bonita de que ves al bebéy dices 'chale, no bay cosa más bonita y frágil, hay que cuidarla, ver por su bienestar '". A partir de ese momento decidió alejarse de las conductas ilegales, "me sali de vender drogas, intenté irme por la derecha, empecé a trabajar".

Hugo refirió que el embarazo "no estaba planeado, pero ya cuando me dijo pues le dije que estaba bien, lo acepté y ella también". Su pareja hubiera preferido esperar más tiempo para embarazarse "ella decía que no, que hasta que creciera un poco más la niña [hija adquirida], pero la cosa se dio (...) pues ya salió embarazada y lo aceptó".

Carlos consideraba que "se iba a componer" si comenzaba a vivir con su pareja "ya llevaba un tiempo con mi pareja viéndonos y todo hasta que yo le dije que me iba a componer juntándome o sea de ya tener alguna responsabilidad, hablamos con su familia y con mi familia para juntarnos". El embarazo "ni fue planeado, pues salió (...) si lo queríamos tener, o sea ya habiamos querido, pero nunca lo habiamos planeado". Para el adolescente la paternidad es "muy bonito, cambias mucho en todo, en tu aspecto, en todo".

\section{Transmisión intergeneracional de la violencia}

Se refiere a la intención del adolescente de no continuar con la transmisión de la violencia que él experimentó en la niñez por parte de su padre, dando cuenta de su propio sufrimiento y de la importancia de proteger a sus hijos de la violencia de familiares cercanos, aunque ello implique tener conductas agresivas.

"Ahi nace de que yo hacia mis hijos ... no quiero que vivan lo que yo vivi, no quiero que sufran lo que yo sufri, quiero darles una mejor educación ya que pues digo, no hay que violentarlos, no hay que hacerlos que entiendan a golpes porque creo que es un problema muy grande se va a haciendo como una cadenita... por eso yo trato de a mis hijos hablarles, platicar con ellos, de tratarlos bien, pero también de hacerlos entender" (Bruno).

Bruno busca que su esposa no ejerza algún tipo de violencia física hacia sus hijos y considera emplearla si es necesario. Asimismo, identifica como legítimo que el varón sea la autoridad de casa y que, si él no la está ejerciendo de forma violenta, su esposa no tiene el derecho de hacerlo, como lo refiere a continuación:

"luego se le pasa la mano con la nalgada y pues yo entre jugando y no ¿verdad? porque digo 'yo que soy el papá y son los que más les pegan a sus bijos y todo ese pedo, yo ni si quiera los toco o sea yo les hablo'; no que 'si', o sea si darles un manazo pero 'es que luego si te manchas' y le digo a veces jugando 'a lo mejor asi te trataba tu madre' y asi como que agarra el pedo y ya le va bajando, o que luego le doy un manazo cuando veo que si se pasa, o 
sea es una forma jugando se podría decir, porque si también llego y le suelto un madrazo lógicamente va haber problemas."

\section{Familia reconstituida}

Se refiere al proceso que atravesaron los adolescentes al asumir un rol parental con su hijo adquirido, y se describen las principales funciones que desempeñan y las expectativas que tienen de la relación con su hijo adquirido. Asimismo, refiere al rol del padre biológico y la postura de la pareja del adolescente ante la duplicación de figuras parentales.

En cuanto a la forma como los adolescentes nombran a su hijo adquirido, se observa cierta ambivalencia, como lo refiere Bruno: "tengo un hijo, otro de los que tengo, pero no es mi hijo, es mi entenado o como les dicen... mi hijastro ¿no?” Lo considera como “hijo de mi esposa, bueno, también mi gordo".

Hugo se expresa de la siguiente forma:

"desde un principio [mi pareja] me dijo que tenía una niña pero yo pensé que no era cierto y cuando me enseñó a la niña, pues ya tuve que aceptarla así (...) desde que nos juntamos yo me hice cargo de la niña". Considera que "al principio sí me costó trabajo [aceptarla] pero ya ahorita ya me acostumbré (...) la veo como mi hija, pero yo sé que tiene a su papá y ella tiene la decisión ahorita porque está chiquita."

La pareja de Hugo lo presentó como su padre "ella desde un principio le dijo a la niña 'ahi está tu papá', o sea a mí. Y la niña, si yo estoy en otro cuarto va y me toca y me dice 'papá'".

Hugo muestra el proceso por el que ha transitado para desarrollar un rol parental con su hija adquirida, pues comenzó con la negación de saber sobre su existencia, después la aceptó y comenzó a tener un rol como proveedor. Hugo se encuentra en espera de que ella crezca y decida a quién nombrará papá, pues actualmente tanto a él como al padre biológico los nombra así, "a míme dice papá y también a él, pero va a crecer y va a decidir". Con relación al rol del padre biológico, Hugo refiere "él no la quiere a ella (...) nunca se ha hecho responsable, nunca ha visto por ellas". A Hugo le interesa que su hija adquirida sepa "que yo me hago cargo de ella". Otra arista más se conforma por el aspecto legal pues ella tiene el apellido "del chavo este, pero estamos esperando a que yo cumpla 18 para cambiar lo del apellido".

Carlos relató que su pareja tiene un hijo previo a su relación de pareja: "desde que me junté ya traía un hijo (...) de 3 años, y asifuimos conviviendo, y ahorita se dedica a él (...) lo quiero como un hijo, aunque él tiene a su papá, yo lo quiero como un hijo". Carlos quiere ser "bien cariñoso con ellos, sacarlos a pasear y hacer lo que no se pudo conmigo pues con ellos". Su hijo adquirido se refiere a él por su nombre y mencionó que "nunca le digo que me diga papá, ahora si que cuando él esté grande (...) si me dijera me sentiría muy orgulloso". El padre biológico "anda muy metido en el vicio, [el hijo adquirido] ha dicho que por qué no lo ve, que si ya no lo quiere y su mamá le dice 'si te quiere, pero ahorita no puede venir'".

En ocasiones Carlos y su pareja han discutido porque "mi esposa se lo deja llevar, pero él anda muy drogado (...) ¿por quélo deja ir? si está viendo 
que su papá, no de que no lo deje ir, es su papá, pero que se ponga a pensar tantito". En este caso, Carlos experimenta malestar porque, aunque él realiza las funciones parentales con su hijo adquirido, el que tiene un lugar privilegiado es el padre biológico.

\section{Discusión}

Se lograron identificar algunos hallazgos principales a partir de la narrativa de las trayectorias de vida de los adolescentes. Por una parte, el experimentar desde la niñez la carencia afectiva, crecer en un contexto de violencia familiar y la muerte de un referente de nutrición emocional. Dichas situaciones se fueron enlazando con factores de riesgo que llevaron al adolescente a ser procesado por la comisión de un delito.

La literatura refiere que cometer delitos en la adolescencia es un factor de riesgo para ser padre joven (Wei, Loeber, \& Stouthamer-Loeber, 2002), y por otra parte, para los adolescentes en situaciones de riesgo ser padre es un reordenamiento de la vida, una forma de construirse un futuro (Fuller, 2000). Lo anterior coincide con los casos analizados ya que a pesar de que el embarazo no fue planeado, los adolescentes lo aceptaron y lo definieron como una oportunidad para alejarse de conductas de riesgo e ilegales.

Identificamos que cuando los adolescentes conforman su propia familia, ésta les ayuda a reconstruir su identidad de no delincuente (Fishman, 1989), es decir, de ser un padre adolescente encargado de velar por el bienestar de sus hijos biológicos y adquiridos.

En su rol parental prevalecían algunas pautas de interacción aprendidas y los roles tradicionales de género en donde el varón tiene mayor jerarquía, se asume como el responsable de mantener el orden en la familia (Andrés, 2004), es el protector de sus hijos y el depositario del poder económico, social y sexual (Villaseñor \& Castañeda, 2003).

En estos relatos se ve a la paternidad como un proceso en el que hay un antes y un después en su trayectoria de vida. Durante la gestación, los hombres se apegan al modelo masculino de ser proveedores y cuidadores de sus parejas (Hernández, 2014). Con el nacimiento de su hijo los padres adolescentes experimentan alegría, afecto, miedo, tensión emocional intensa (Rodríguez, 2009) y algunas de las características de sus hijos se las atribuyen a sí mismos (Botero \& Castrillón, 2015). Después del nacimiento, los varones sienten la responsabilidad de cuidar el desarrollo de sus hijos. La paternidad es un proceso en el que la identidad de los participantes se va construyendo y reconstruyendo (Ortega, Torres, \& Salguero, 2009) a través de asumir responsabilidades parentales y reparar intergeneracionalmente sus propias infancias (Tuffin et al., 2010).

Un evento que observamos fue la intención de detener la transmisión intergeneracional de la carencia afectiva, la cual se caracteriza por una cultura afectiva y relacional cargada de eventos carenciales, no elaborados e inexpresados, lo cual se convierte en un obstáculo para una adecuada asunción del rol paterno (Cirillo, Berrini, Cambiaso, \& Mazza, 1999). Identificar familiares que compensen las carencias de nutrición emocional 
se vuelve un elemento importante para que ésta no tenga carácter de irreversible fatalidad (Linares, 1996).

Uno de los hallazgos de la investigación es que existía el riesgo de que se continuara la transmisión intergeneracional de la violencia familiar experimentada en la niñez y que continuaba en la adolescencia. Smith et al. (2005) consideran que el maltrato en la adolescencia por parte de sus padres incrementa el número de arrestos, delitos y consumo de drogas en los años posteriores. Lo anterior fue corroborado en el caso de un adolescente testigo y receptor de violencia por parte de su padre desde la niñez, y en la adolescencia buscó una forma de detenerla; sin embargo, dichas soluciones eran conductas de riesgo como consumir drogas.

En el relato de Bruno se muestra una posición contradictoria porque, por una parte, trata de "detener la cadenita" de violencia y por otra parte repite la conducta violenta con su esposa, considerándola como una forma de detener la violencia de su pareja hacia sus hijos. Se ha identificado que, si un niño vive en un ambiente violento, su desarrollo se ve afectado y es posible que repitan comportamientos similares en sus propias familias (Ruiz, 2000). Asimismo, las relaciones familiares caracterizadas por la hostilidad, irritabilidad y rechazo guardan una relación positiva con el comportamiento agresivo (Mestre et al., 2004).

La repetición de patrones de conducta indeseados, entre ellos el abuso que se repite y perpetúa, es posible debido a que los protagonistas "no ven que no ven" y siguen una lógica que ellos perciben como coherente, lo que les impide tomar conciencia del significado y de las consecuencias perjudiciales de sus propias conductas (Ravazzola, 2003). Esto puede suceder con Bruno, pues con la intención de que sus hijos no sean violentados, él puede llegar a agredir a su pareja para que ella no los violente.

Bruno ha reflexionado acerca de no repetir la violencia con sus hijos, es decir, busca ser un referente afectivo, en donde no experimenten patrones desadaptativos violentos, y así sus hijos tendrán la posibilidad de no repetirlos y lograr establecer relaciones sociales más funcionales (ParraCardona et al., 2008).

Uno de los aspectos centrales en las familias reconstituidas de los adolescentes es la presencia de su hijo adquirido. Los adolescentes se cuestionan a quién va a nombrar padre, ¿al papá adquirido, al papá biológico o a ambos? Se identificó que la principal forma como los padres adolescentes explican y legitiman su lugar en la familia reconstituida es desde su rol de proveedores, y de forma secundaria buscan cercanía con sus hijos; con los biológicos y los adquiridos. El rol parental respecto al hijo adquirido se basa en la sustitución (Jociles \& Villaamil, 2008), que se caracteriza por ocupar el lugar del padre biológico, se basa en una imagen tradicional de la familia, como si buscaran encajar en ser una familia nuclear, suplen al padre biológico debido a que éste no tiene un rol parental estable o es totalmente ausente (Rivas, 2012). Es decir, no existe una multiplicación de las figuras parentales pues el adolescente asume la función parental (Fruggeri, 2005). 
Coincidimos con Papernow (2018), quien afirma que convertirse en una familia reconstituida es un proceso y no un evento, por lo que requiere tiempo, un buen mapa del territorio y algunas indicaciones de manejo precisas.

Entre las limitaciones del estudio se encuentra el reducido número de participantes y que no se incluyeron en las entrevistas a las parejas de los adolescentes, quienes podrían enriquecer los resultados y aportarían una mirada diferente de la paternidad de los participantes.

\section{Conclusiones}

El presente análisis tuvo como objetivo examinar la forma como los adolescentes en conflicto con la ley construyen su paternidad, específicamente en relación a sus experiencias en su familia de origen, la conducta delictiva y al tener una familia reconstituida en México. A manera de conclusión identificamos la importancia de mirar a las familias desde sus múltiples posibilidades. Específicamente esta población en la que las investigaciones se han enfocado en el déficit o irresponsabilidad como padres y hasta hace poco tiempo se está considerando la contraparte, es decir, la paternidad como una elección y como punto de inflexión.

El estudio es una contribución al estudio de la paternidad, no solo biológica sino también adquirida. Lo anterior implica un elemento de mayor complejidad al brindar tratamiento psicoterapéutico a los padres adolescentes en conflicto con la ley que se encuentran en proceso de reinserción social, por lo que este estudio puede sentar las bases para que, en los programas de atención psicoterapéutica, se visibilice la paternidad en familias reconstituidas en esta población.

Respecto a las familias reconstituidas coincidimos con Fruggeri (2001), acerca de que la pregunta que se hace la investigación científica no es si las familias diferentes a la nuclear son capaces de satisfacer las funciones, sino ¿cómo lo hacen? Es importante tener en cuenta que una segunda unión no sustituye la unión anterior, sino que se interconectan pues hay vínculos que se han articulado en torno a ella y a través de ella.

Estudiar a las familias reconstituidas es una oportunidad para analizar cuestiones como el rol que desempeñan los miembros de la familia, porque este tipo de familias requiere de mayor flexibilidad para asumir los roles y funciones (Fruggeri, 2005).

Para futuras investigaciones se recomienda continuar con estudios que permitan comprender el complejo universo de la paternidad en adolescentes y, específicamente, aquellos en conflicto con la ley, desde una perspectiva sistémica. También realizar entrevistas con algún miembro significativo de su familia de origen y su pareja para profundizar en la comprensión del proceso de construcción de su paternidad y así ampliar las posibilidades de intervención en la práctica clínica. Coincidimos con Ganong y Coleman (2018) acerca de la importancia de contribuir con el estudio de la resiliencia de las familias reconstituidas, lo cual se puede hacer a través de explorar la familia de origen (Papernow, 2018). 


\section{Referencias}

Andrés, P. (2004). Violencia contra las mujeres, violencia de género. En Q. RuizJarabo \& P. Blanco (Eds.), La violencia contra las mujeres. Prevención y detección. Cómo promover desde los servicios sanitarios relaciones autónomas, solidarias y gozosas (pp. 17-38). Madrid, España: Díaz de Santos.

Balam, J. M., Valdez, A. G., Ramírez, A., \& Padilla, N. (2018). Significado psicológico, roles y expectativas de la paternidad en adolescentes mexicanos: Estudio exploratorio. Psicología Iberoamericana, 26(1), 42-52.

Botero, L., \& Castrillón, L. (2015). La experiencia de la paternidad en adolescentes. Revista Virtual Universidad Católica Del Norte, 46, 89-101. Recuperado de http://revistavirtual.ucn.edu.co/index.php/RevistaUCN /article/view/701/1228

Chavarín-García, M. C., \& Gálvez-Hernández, C. (2018). Conducta antisocial adolescente y dinámica familiar. Análisis conceptual. Psicología Iberoamericana, 26(1), 1-13.

Cirillo, S., Berrini, R., Cambiaso, G., \& Mazza, R. (1999). La familia del toxicodependiente. Barcelona, España: Paidós.

De Jesús-Reyes, D., \& Cabello-Garza, M. L. (2011). Paternidad adolescente y transición a la adultez: Una mirada cualitativa en un contexto de marginación social. Iberofórum Revista de Ciencias Sociales de la Universidad Iberoamericana, 6(11), 1-27.

De Keijzer, B. (2003). Hasta donde el cuerpo aguante: Género, cuerpo y salud masculina. En C. Caceres, M. Cuerto \& M. Ramos (Eds.), La salud como derecho ciudadano: perspectivas y propuestas desde América Latina. Lima, Perú: Facultad de Salud Pública y Administración de la Universidad Peruana Cayetano Herida.

Fishman, C. (1989). Tratamiento de adolescentes con problemas. Un enfoque de terapia familiar. Buenos Aires, Argentina: Paidós.

Franzoni, J. (2014). Factores que inciden en la participación de los hombres en la crianza de los hijos. En A. Figueroa, J. \& Salguero (Eds.), ¿Y si hablas de...sde tu ser hombre? Violencia, paternidad, homoerotismo yenvejecimiento en la experiencia de algunos varones (pp. 271-302). CDMX, México: El Colegio de México.

Fruggeri, L. (2001). Los conceptos de mononuclearidad y plurinuclearidad en la definición de familia. Revista Del Centro Milanese Di Terapia Della Famiglia, 8, 11-22.

Fruggeri, L. (2005). Diverse normalita: Psicologia delle relazionifamiliari. Roma, Italia: Carocci Editore.

Fruggeri, L. (2016). Diferentes normalidades. Psicología social de las relaciones familiares. México: Editorial Página seis.

Fuller, N. (2000). Paternidades en América Latina. Lima, Perú: Fondo Editorial 2000.

Ganong, L., \& Coleman, M. (2018). Studying stepfamilies: Four eras of family scholarship. Family Process, 57(1), 7-24. https://doi.org/10.1111/famp. 12307

Hernández, A. (2014). Representación social de la paternidad y significado de la progenie en jóvenes que viven en la calle. En J. Figueroa \& A. Salguero (Eds.), ¿Y si hablas de...sde tu ser hombre? Violencia, paternidad, 
homoerotismo y envejecimiento en la experiencia de algunos varones (pp. 237-270). CDMX, México: El Colegio de México.

Honorable Congreso de la Unión. (2007). Ley de justicia para adolescentes para el distrito federal. CDMX, México: Gaceta Oficial del Distrito Federal.

Hsieh, H. F., \& Shannon, S. E. (2005). Three approaches to qualitative content analysis. Qualitative Health Research, 15(9), 1277-1288. https://doi.org /10.1177/1049732305276687

Instituto Nacional de Estadística y Geografía (INEGI). (2015). Encuesta intercensal 2015. CDMX, México: INEGI.

Jensen, T. M. (2019). A typology of interactional patterns between youth and their stepfathers: Associations with family relationship quality and youth well-being. Family Process, 58(2), 384-403. https://doi.org/10.1111/fam p. 12348

Jociles Rubio, M. I., \& Villaamil Pérez, F. (2008). Estrategias de sustitución en la construcción de la paternidad y la maternidad dentro de las familias reconstituidas. Papers: Revista de Sociologia, 90, 213-240. https://doi.org /10.5565/rev/papers/v90n0.743

Landers, M. D., Mitchell, O., \& Coates, E. E. (2015). Teenage fatherhood as a potential turning point in the lives of delinquent youth. Journal of Child and Family Studies, 24(6), 1685-1696. https://doi.org/10.1007/s10826 $-014-9971-\mathrm{y}$

Linares, J. (1996). Identidad y narrativa. La terapia familiar en la práctica clínica. Barcelona, España: Paidós.

López, J. R., \& López, C. (2008). Conductas antisociales y delictivas en la adolescencia. Murcia, España: Editum Salud.

Lykken, D. (2000). Las personalidades antisociales. Barcelona, España: Gedisa.

Mallimaci, F., \& Giménez, V. (2006). Historia de vida y métodos biográficos. En I. Vasilachis (Ed.), Estrategias de investigación cualitativa (pp. 175-209). Barcelona, España: Gedisa.

Mestre, V., Samper, P., \& Frías, D. (2004). Personalidad y contexto familiar como factores predictores de la disposición prosocial y antisocial de los adolescentes. Revista Latinoamericana de Psicologia, 36(3), 445-457.

Ngu, L., \& Florsheim, P. (2011). The development of relational competence among young high-risk fathers across the transition to parenthood. Family Process, 50(2), 184-202. https://doi.org/10.1111/j.1545-5300.2011.013 54.x

Olvera, X. A. (2012). Embarazo adolescente y madres jóvenes en México. México: Secretaría de Educación Pública. https://doi.org/9786079200633

Ortega, P., Torres, L. E., \& Salguero, A. (2009). Paternidad: Periodo de cambio en la vida de los varones. Revista PsicologiaCientifica.Com, 11(21).

Papernow, P. L. (2018). Clinical guidelines for working with stepfamilies: What family, couple, individual, and child therapists need to know. Family Process, 57(1), 25-51. https://doi.org/10.1111/famp.12321

Parra-Cardona, J. R., Sharp, E. A., \& Wampler, R. S. (2008). "Changing for my kid": Fatherhood experiences of Mexican-origin teen fathers involved in the justice system. Journal of Marital and Family Therapy, 34(3), 369-387. https://doi.org/10.1111/j.1752-0606.2008.00078.x

Ravazzola, M. (2003). Historias infames: Los maltratos en las relaciones. Buenos Aires, Argentina: Paidós. 
Rivas, A. (2012). El ejercicio de la parentalidad en las familias reconstituidas. Portularia, 12(2), 29-41.

Rodríguez, G. (2009). La paternidad en el adolescente: Un problema social. Archivos Venezolanos de Puericultura y Pediatría, 72(3), 86-91.

Rodríguez, G., Gil, J., \& García, E. (1999). Metodología de la investigación cualitativa. Granada, España: Aljibe.

Ruiz, P. (2000). Comentarios a las ponencias de Benno de Keijzer, de Javier Alatorre y Rafael Luna. En N. Fuller (Ed.), Paternidades en América Latina (pp. 277-288). Lima, Perú: Fondo Editorial 2000.

Salguero, V. M. (2006). Significado y vivencia de la paternidad en algunos varones de los sectores socioeconómicos medios en la Ciudad de México. En O. Figueroa, \& J. G. Tena (Eds.), Ser padres, esposos e hijos: prácticas $y$ valoraciones de varones mexicanos (pp. 57-94). CDMX, México: El Colegio de México.

Shannon, S. K. S., \& Abrams, L. S. (2007). Juvenile offenders as fathers: Perceptions of fatherhood, crime, and becoming an adult. Families in Society, 88(2), 183-191. https://doi.org/10.1606/1044-3894.3616

Shepard, B. (2000). Comentarios a las ponencias de Norma Fuller, Mara Viveros y José Olavarría. En N. Fuller (Ed.), Paternidades en América Latina (pp. 175-190). Lima, Perú: Fondo Editorial 2000.

Smith, C. A., Ireland, T. O., \& Thornberry, T. P. (2005). Adolescent maltreatment and its impact on young adult antisocial behavior. Child Abuse and Neglect, 29(10), 1099-1119. https://doi.org/10.1016/j.chiab u.2005.02.011

Stern, C. (2004). Vulnerabilidad social y embarazo adolescente en México. Papeles de Población, 39(10), 129-158.

Thornberry, T., Wei, E., Stouthamer-Loeber, M., \& Van Dyke, J. (2000). Teenage fatherhood and delinquent behavior. Juvenile Justice Bulletin. Washington, DC, Estados Unidos: Office of Juvenile Justice and Delinquency Prevention.

Tuffin, K., Rouch, G., \& Frewin, K. (2010). Constructing adolescent fatherhood: Responsibilities and intergenerational repair. Culture, Health and Sexuality, 12(5), 485-498. https://doi.org/10.1080/136910510036 29359

Vela, F. (2008). Un acto metodológico básico de la investigación social: La entrevista cualitativa. En M. Tarrés (Ed.), Observar, escuchary comprender. Sobre la tradición cualitativa en la investigación social (pp. 63-91). México: Miguel Ángel Porrúa.

Villaseñor, M., \& Castañeda, J. (2003). Masculinidad, sexualidad, poder y violencia: Análisis de significados en adolescentes. Salud Pública de México, 45(1), 44-57.

Wei, E. H., Loeber, R., \& Stouthamer-Loeber, M. (2002). How many of the offspring born to teenage fathers are produced by repeat serious delinquents? Criminal Behaviour and Mental Health, 12(1), 83-98. http s://doi.org/10.1002/cbm.488 